\title{
Marcel Jousse : à la croisée de l'anthropologie et des neurosciences, le rythme des corps
}

Denis Cerclet

\section{(2) OpenEdition \\ 12 Journals}

Édition électronique

URL : http://journals.openedition.org/pa/310

DOI : 10.4000/pa.310

ISSN : 2273-0362

Éditeur

Université Lumière Lyon 2

Édition imprimée

Pagination : 24-38

ISBN : 1634-7706

ISSN : $1634-7706$

\section{Référence électronique}

Denis Cerclet, « Marcel Jousse : à la croisée de l'anthropologie et des neurosciences, le rythme des

corps », Parcours anthropologiques [En ligne], 9 | 2014, mis en ligne le 30 septembre 2014, consulté le 19 avril 2019. URL : http://journals.openedition.org/pa/310 ; DOI : 10.4000/pa.310

Parcours anthropologique 


\section{Marcel Jousse : à la croisée de l'anthropologie et des neurosciences, le rythme des corps}

\section{Denis Cerclet}

Université Lumière Lyon 2, CREA

Longtemps les sciences sociales se sont intéressées à l'individu comme à un atome. La société est dès lors un jeu d'assemblage et de distinction. Mais c'est oublier la part d'indivision, le lien continuel qui les relie et qui interroge la distinction société/individu (Elias, 1991). La société peut être comprise comme un mouvement global et pourtant localisé qui se perpétue parce que les individus se réalisent eux-mêmes, ensemble dans un flux ininterrompu.

Voici quarante ans que paraissaient, à titre posthume, trois textes de Marcel Jousse sous l'intitulé L'Anthropologie du geste. Cet intérêt pour le corps était novateur et l'œuvre mérite aujourd'hui d'être relue. Je me propose de mettre en relation la lecture que j'ai faite de la pensée de Marcel Jousse avec une ethnographie de certains travaux actuels en neuroscience. Il me semble utile de faire ces liens à la fois pour actualiser une démarche que je rapprocherais volontiers de celle de Maurice Merleau-Ponty et insister sur l'importance du rôle que peut jouer l'œuvre de Jousse dans le renouvellement des sciences humaines et sociales.

L'anthropologie de Jousse est fondamentalement dynamique. Elle prend le contrepied $\mathrm{du}$ discours anthropologique ambiant de son époque en s'appuyant sur les avancées scientifiques d'autres disciplines telles la psychiatrie, la médecine, la phonétique et la dialectologie. Marcel Jousse change délibérément de paradigme et, s'intéressant à l'étude de l'homme corporé et des modalités physiques et biologiques du social, il inscrit pleinement les sciences humaines et sociales dans le domaine des sciences du vivant.

Le modèle développé par Marcel Jousse repose sur la présence de l'homme dans le cosmos qui est "ordre et ordonnance ", imbrication ou complexus d'interactions. Nous pouvons dire que l'homme est situé, aussi bien à l'échelle globale que locale, du cosmos ou du pays. Etre paysan c'est être informé par son pays. Cet homme est inséparable de son environnement sauf à courir le risque d'être "dépaysannisé », dépaysé, "algébrosé », porté à pratiquer un langage désincarné.

C'est l'homme, l'Anthropos, qui confère à ce système global sa dimension anthropique parce qu'il se comporte comme révélateur, «vivant résonateur ». Homme qui, lui-même, ignore « ce qui va être sa substance » (1974:526, note 
5), qui n'a pas de soi proprement dit : il ne prend conscience de lui-même qu'à travers les interactions. Il est, par nature, moteur, motricité. Et il est perçu, "mimé comme un geste qui lui est propre » (idem : 52), saisi d'une manière si particulière que cela équivaut à le nommer. Ainsi, Jousse déclare: «De l'anthropologie statique, nous entrons dans l'anthropologie dynamique. A un géométrique alignement de squelettes se substitue la richesse d'êtres vivants » (ibid. : 721, note 2).

Ce modèle est fondamentalement relationnel, indéfini. «Les corps attirent les corps ». Rien ne les sépare et les phénomènes de résonance vont les inscrire à la fois dans l'absorption d'autrui - rendue possible par la non-connaissance de soi - et la restitution. Il y a diffusion d'énergie selon une logique vibratoire, une mécanique ondulatoire, une dynamique interactionnelle.

L'être humain saisit le monde extérieur et l'incorpore «l'intussusceptionne » - pour coïncider avec les actions qui proviennent de son environnement. Et, par un effet du vivant, quasi physique, l'être humain va gestualiser ce qui lui a été infligé. C'est le Mimisme qui « est la tendance instinctive que seul possède l'anthropos à "rejouer" les gestes du réel qui se sont "joués" devant lui. Cette grande force contraignante, nous l'avons dès que nous nous éveillons à la vie » (ibid. : 691, note 14). Entre le « Jeu», comme impression et le « Rejeu » comme expression, il n'y a pas qu'un seul transfert d'énergie. Certes l'Agent Agissant l'Agit mais l'être humain « intellige » les interactions du réel. Il a la capacité de composer, décomposer, recomposer les actions et les gestes dans un « rejeu » de ce qui s'est joué en lui. Il n'y a pas d'intelligence ni de connaissance, ni même de mémoire sans cette gestualisation, qu'elle soit de tout le corps (corporage) ou des mains (manuelage) ou encore de l'appareil laryngo-buccal (langage). "On ne comprend vraiment que ce que l'on rejoue » (ibid. : 962); il n'y a pas de connaissance du réel hors de ce qui se passe en nous.

La mécanique humaine «est, elle aussi, une mécanique ondulatoire. Elle se joue par ondes, par vagues, par phases rythmiques d'interaction » (ibid. : 149). Certes Jousse prend soin de définir le rythme comme «le retour d'un même phénomène anthropologique à des intervalles biologiquement équivalents » (ibid. : 146) mais il précise bien que cette scansion n'est jamais mathématiquement métrique. Nous percevons de manière rythmée, par vagues successives. Nous sommes rythmés et rythmisants. Sur le plan biologique, il y a le rythme des organes, celui du pas, ou du balancement des mains. Et nous rythmisons parce que nous successivons selon le rythme d'un flux musculaire. Nous ne morcelons pas : «Le Rythmisme va nécessairement distribuer et successiver vitalement le Mimisme " (ibid. : 53). Le temps n'est pas fait d'instants et le mouvement est continu. Et si nous avons l'impression de distinguer les mots, les poses successives d'un geste, c'est ou bien par facilité ou par algébrosie.

Jousse a introduit le corps en anthropologie. Non pas un corps objet, dont nous pourrions nous distinguer. Le corps de Jousse est un corps vivant, qui se 
balance, qui ondule rythmiquement sur la base de compétences telles le mimisme et l'intussusception. La vie humaine repose sur tout un ensemble de gestes corporels, manuels, oculaires, auriculaires, laryngo-bucaux, gustatifs, olfactifs et tactiles. Ce corps, loin de se réduire au squelette, est un « instrument souple qui va suivre toute la fluidité du réel. Instrument qui va pouvoir rejouer le monde tout entier » (ibid. : 57).

Cette perspective est "révolutionnaire " dans le sens où elle réconcilie le corps et la pensée, où elle replace l'individu dans le monde, fait communiquer l'intérieur et l'extérieur et se porte à l'écoute des sensations, des émotions et des perceptions. Tout se tient et vibre d'une même totalité.

L'anthropologie de Jousse fait écho à certains travaux qui relèvent d'autres disciplines telles la philosophie ou les neurosciences.

Je vais aborder certaines de ces approches qui traitent du corps, de la résonance, de la synchronisation et du rythme. Nous parviendrons, je l'espère à reconnaître une configuration relativement proche de celle de Jousse. Il ne s'agit pas d'actualiser la pensée de Jousse mais plutôt de revenir à la fois sur l'actualité de l'édifice qu'il a construit et le situer dans ce que l'on pourrait reconnaître comme une filiation théorique.

\section{AU CEEUR DE LA RELATION MOI-AUTRUI-LE MONDE, LE GESTE}

La question du corps revêt aujourd'hui une importance d'autant plus grande qu'elle fut tenue à l'écart. Autrefois lieu du débordement, de la transgression de la norme, de l'animalité, le corps retrouve désormais une place centrale, à tel point sans doute qu'il phagocyte l'esprit.

Richard Shusterman s'élève contre les théories selon lesquelles il n'y aurait de médiation avec le réel que langagière. Critiquant le tout interprétation, il relève que la compréhension peut passer par des signes modestes et pas seulement par des interprétations : "une réaction particulière, un frémissement, un petit geste, peuvent suffire à indiquer que l'on a compris » (1994: 75). Il s'adresse aux philosophes et leur déclare : "Si nous ne comprenons pas cela, nous autres philosophes, c'est parce que pour les têtes parlantes et désincarnées que nous sommes, la seule forme d'expérience que nous reconnaissons et légitimions est de nature linguistique : penser, parler, écrire. Mais ni le langage, qui contribue à nous former, ni nous-mêmes ne pourrions survivre sans l'arrière-plan inexprimé d'expérience et de compréhension préréflexives et non linguistiques qui en constitue la condition » (idem : 67-68).

Poursuivant son cheminement vers une meilleure intégration du corps en philosophie, Richard Shusterman développe ce qu'il appelle la soma esthétique. Le corps de Shusterman n'est pas seulement le corps objet, physique (j'ai un corps), c'est aussi le corps de «Je suis corps » et le corpsesprit, le body-mind de John Dewey. Il reconnaît que le corps est le medium 
fondamental de notre relation au monde : il est au cœur de la perception, de l'action et de la pensée. Dans le cadre de son approche pluridisciplinaire, il utilise le terme soma pour désigner le nœud où s'intègre le corps, l'esprit, la cognition, le social, le politique et la dimension esthétique propre à la vie quotidienne. "Soma sensoriel et sensible plutôt que simple cadavre mécanique » (Shusterman, in Formis, $2009: 45)$. Le soma c'est tout à la fois des os, des connexions neuronales, des habitudes, des comportements, des statuts et des fonctions sociales. Le champ de la soma-esthétique est « l'étude critique et la culture méliorative de notre expérience et de notre usage du corps vivant en tant que site d'appréciation sensorielle et de façonnement créateur de soi » (Shusterman, 2007 : 11). Le terme esthétique dit l'importance que Shusterman attache à la fois à la perception, à l'appréciation d'autres que soi et à la stylisation de soi. Il pense la soma-esthétique comme une discipline car elle vise autant à comprendre la place que prend le corps-esprit dans le monde spatio-temporel qu'à aiguiser les compétences perceptives dans la perspective d'améliorer l'expérience. Shusterman se réfère à une intelligence somatique qui, bien que partiellement «spontanée, non réfléchie, $[. .$.$] n'en demeure pas$ moins fondée sur des compétences acquises qui expriment une intelligence alors même qu'il n'y a pas de réflexion conceptuelle et de délibération » (Soulez, $2010: 8$ ).

Maurice Merleau-Ponty a marqué de son empreinte ce champ de recherche par sa manière de penser le monde comme une totalité signifiante pour l'homme en acte. Il met en évidence l'inséparabilité de ces trois termes «moiautrui-le-monde » qui se réalise comme un système grâce à l'expérience, la perception et la conscience humaine. "Le corps est le véhicule de l'être au monde, et avoir un corps c'est pour un vivant se joindre à un milieu défini, se confondre avec certains projets et s'y engager continuellement » (MerleauPonty, 1945 : 97). L'être humain n'établit pas une relation avec autrui avec des représentations ou une pensée explicite: " je ne comprends pas les gestes d'autrui par un acte d'interprétation intellectuelle. [...] C'est par mon corps que je comprends autrui, comme c'est par mon corps que je perçois des "choses" » (idem : 216). Cet individu communique avant tout avec "un sujet parlant » sur un mode particulier qui met en œuvre un certain style d'être, " une modulation synchronique de ma propre existence, une transformation de mon être » (ibid. : 214). Selon Merleau-Ponty, «[t]out se passe comme si l'intention d'autrui habitait mon corps ou comme si mes intentions habitaient le sien » (ibid. : 215).

L'organisme est inséré dans une situation particulière, selon une configuration particulière, et dans des conditions écologiquement situées. Selon Francisco Varela, « [1] cerveau existe dans un corps, le corps existe dans le monde, et l'organisme agit, bouge, chasse, se reproduit, rêve, imagine. Et c'est de cette activité permanente qu'émergent le sens de son monde et les choses » (in Kempf, 1998 : 109). Pour rendre compte de cette approche, on parle de cognition incarnée, ou encore d'énaction un néologisme introduit par 
Varela. Ainsi, cette citation de l'ouvrage qu'il a publié avec Thompson et Rosch :

Par le mot incarné, nous voulons souligner deux points : tout d'abord, la cognition dépend des types d'expériences qui découlent du fait d'avoir un corps doté de diverses capacités sensori-motrices; en second lieu, ces capacités individuelles sensorimotrices s'inscrivent elles-mêmes dans un contexte biologique, psychologique et culturel plus large. En recourant au terme action, nous souhaitons souligner une fois de plus que les processus sensoriels et moteurs, la perception et l'action sont fondamentalement inséparables dans la cognition vécue. (1991 : 234)

Les approches classiques reposent sur un modèle selon lequel le système sensoriel informe le système cognitif et le système moteur effectue la décision $\mathrm{du}$ système cognitif. Alors que dans le cadre des théories fondées sur l'incarnation, le comportement humain et son attachement à l'environnement relèvent d'un lien indéfectible entre le système sensorimoteur, la cognition et les émotions.

Les auteurs sont devenus nombreux à défendre le principe d'une coévolution des organismes et de l'environnement, tablant sur un échange incessant entre les processus internes des organismes et le monde extérieur. Bernard Andrieu, qui explore de nombreuses approches du corps, parle de «monde corporel»: «le monde corporel n'est ni le monde ni le corps. Le corps a incorporé le monde par l'apprentissage au point de se mondaniser par habitus dans ses fonctions et ses modes d'action tactile. [...] Cet art plastique de soi rend le corps mondain et le monde corporel » $(2010: 68)$.

Pour accentuer cette proximité entre l'être humain et son environnement, Alain Berthoz s'affranchit de la notion de représentation au profit d'une théorie fondée sur la notion d'acte ou d'action. « Le but n'est pas de "retrouver le corps", d'en faire une annexe importante mais secondaire de la raison. Le but est bien de renverser le propos et de partir du corps agissant. [...] L'acte ou le geste ne se réduisent pas à des muscles. Pour moi, l'action n'est pas la motricité : l'action et le geste sont projets, intentions, émotions, souvenirs. » Le cerveau humain « est une partie du monde, qui en a internalisé les propriétés et en émule certaines mais les réfère à ses propres buts » (2003 : 169).

Rudolfo Llinas apporte une contribution intéressante qui permet d'approfondir cette idée et d'organiser la relation entre soi et le monde autour du mouvement et du geste: "La génération centrale du mouvement et la génération de la pensée sont profondément connexes; elles sont en fait différentes parties du même processus. De mon point de vue, [...] la pensée est l'intériorisation du mouvement » (2001 : 5). Le langage devient geste et se libère de son référencement à l'esprit et à l'abstraction conceptuelle. Nombreux sont les auteurs qui associent la naissance du langage aux gestes de la vie quotidienne et à une communication par le toucher. Le langage serait «un genre spécial de motilité orale»(Gallagher, 2005). Selon Andrew N. 
Meltzoff « $[\mathrm{u}] \mathrm{n}$ acte humain n'est pas simplement un vecteur de mouvement ou une partie isolée du corps, mais plutôt une transformation finalisée d'un organe » (2002: 52).

Shaun Gallagher s'appuie sur les travaux du psychologue David McNeill qui a montré que les gestes que nous employons quand nous parlons sont synchronisés avec précision avec le discours. Cela laisse penser que les gestes et la parole forment ensemble un système simple et intégré. "Les gestes sont des mouvements qui se produisent seulement pendant la parole; ils sont synchronisés avec les unités linguistiques, sont parallèles dans les fonctions sémantiques et pragmatiques aux unités linguistiques synchronisées; ils remplissent des fonctions du texte comme la parole »(McNeil cité par Gallagher, 2005 : 118-119).

Si nous revenons à l'affirmation de Llinas, «ce que nous appelons pensée est l'internalisation du mouvement, au cours de l'évolution » (2001: 35), les gestes peuvent apparaître comme autant de signes de la construction spatiale de la pensée et du discours. Comme si le corps effectuait un parcours, sur le mode de l'escalade, pour enchaîner, relier les arguments du récit éparpillés en différents espaces du cerveau. Le mouvement du corps indique ces déplacements cognitifs et surtout l'engagement de tout le corps dans cette opération de composition du discours.

Le rapport entre langage et gestes ne relève pas seulement d'un mode de synchronisation interne. L'un et l'autre servent à établir et à entretenir des liens avec autrui. Le geste et le langage semblent avoir une lointaine fonction sociale que certains rapprochent du toilettage et de l'épouillage. Pour Michael Corballis « [1]e toilettage est certainement une forme sociale de communication; il implique une certaine capacité d'adopter la perspective mentale d'autres et peut même être considéré comme un exemple d'altruisme réciproque [...]. Dumbar pointe l'importance, dans la société humaine, du bavardage, qu'il considère comme une forme de toilettage» (2002: 209). Ce lien entre l'environnement pensé sur le mode du geste a aussi été abordé par Maurice Merleau-Ponty. Ainsi, dans Le visible et l'invisible, il affirme que «la vision est palpation par le regard » $(1964: 173)$; «le regard [...] enveloppe, palpe, épouse les choses visibles. [...] tout mouvement de mes yeux - bien plus, tout déplacement de mon corps - a sa place dans le même univers visible que par eux je détaille et j'explore, comme, inversement, toute vision a lieu quelque part dans l'espace tactile » (idem : 175). Poursuivant dans la même voie, Alva Noé considère que la vision est comme le toucher, c'est un mouvement, une exploration de l'environnement. "Comme le contact, la vision est active» (2005: 73). Il nous dit que notre perception n'est pas immédiate, que nous déplaçons les yeux comme nous déplacerions les doigts en aveugle pour identifier une forme, une matière. La perception est soumise à une certaine forme d'habileté comme le toucher.

De nombreux auteurs insistent sur la dimension innée de l'intérêt pour autrui, de l'échange et du partage. Des travaux sur les nouveaux nés mettent 
en évidence la précocité des échanges avec la mère et les proches. Et certains montrent comment cet intérêt ne se porte pas sur les objets à moins qu'ils ne remplissent certaines conditions parmi lesquelles la capacité de répliquer et de rechercher à son tour le lien ainsi qu'une certaine qualité de mouvement. Le nouveau-né semble immédiatement communiquant et à même de s'insérer dans un autre processus que lui-même. Nicolas Georgieff évoque une «pulsion ou mouvement de partage» (2005: \$24) et présente le cerveau comme un organe social, de groupe. Andrew N. Meltzoff table sur un " appariement transmodal entre les actes des autres qui sont vus et les actes produits par soi-même. Ce ne sont pas les caractéristiques statiques des adultes - leurs yeux, leurs cheveux, etc. - qui sont spéciales pour les bébés ; mais la façon dont le corps de l'adulte bouge et ses relations avec soi » (2002: 35).

\section{LES PHÉNOMÈNES DE RÉSONANCE}

La notion de résonance s'est imposée pour tenter de saisir plus précisément les modalités de l'individuation. Cette résonance implique une très grande proximité, si ce n'est le partage des mêmes réseaux cérébraux par l'action, la perception et l'exécution des tâches. Selon Jean Decety,

[1]a correspondance entre action observée et réalisée peut être métaphoriquement décrite en terme de résonance. Cette résonance chez l'observateur ne produit pas nécessairement un mouvement ou une action mais pourrait servir à d'autres fonctions comme activer, à un niveau infra-conscient, l'expérience subjective (avec sa valence affective et émotionnelle) qui serait associée à la génération de l'action perçue. $(2002: 124)$

Ce lien entre perception et action - que l'on désigne par l'expression résonance motrice - a été renforcé avec la découverte des neurones miroirs par l'équipe de Giacomo Rizzolatti (2008) à l'université de Parme. Ces neurones, situés dans le cortex prémoteur, entrent en activité chez une personne lorsqu'elle effectue une action, l'observe chez autrui et l'imagine. Ils ne codent pas de mouvements particuliers mais des actes moteurs c'est-à-dire des actes intentionnels et orientés vers un but. Les gestes, agis ou imaginés, activent ce que Marc Jeannerod (1994) appelle une imagerie motrice qui relie perception du mouvement et préparation à l'action. Ainsi, Filippo Fimiani déclarait que « les gestes, ainsi que les mouvements d'objets agis et dits, en tant qu'actes signifiants, déclenchent automatiquement des réactions imitatives posturales et corporelles, viscérales et motrices, finalement empathiques, et pas seulement visuelles » $(2009: 5)$.

Ce domaine des résonances recouvre plusieurs types de relations au contours pas toujours très clairs parmi lesquels figurent la contagion émotionnelle, la sympathie, l'empathie ou encore la théorie de la simulation. 
L'imitation est un phénomène bien étudié et certains la considèrent comme une matrice comprenant les autres formes de résonance. Marc Jeannerod mettait en évidence que l'imitation est un couplage de la perception et de l'action. Arnaud Revel et Jacqueline Nadel (2007) la considèrent comme innée et la définissent en quatre points : elle requiert la présence d'un partenaire, l'attention partagée, la synchronisation du tempo et l'alternance des rôles entre l'imitateur et l'imité. Ils constatent que l'imitation change l'état d'esprit de chacun des partenaires. Et ils insistent sur la synchronisation et le turntaking (ou changement de rôle) ainsi que sur la capacité des individus à produire et à reconnaître un rythme.

Meltzoff montre comment l'imitation «devient importante pour l'intersubjectivité, parce que c'est la première opportunité pour les bébés d'établir un lien entre le monde visible des autres et leurs propres états internes » (2002: 52). L'imitation ne consiste pas à reproduire fidèlement les gestes mais plutôt à répliquer un schème ou un programme d'action en vue $\mathrm{d}$ 'obtenir un certain résultat. Le but est manifestement la coordination sociale, attirer l'attention, confirmer la présence d'un partenaire, explorer et partager le comportement d'autrui. Effectivement, si l'on se place du point de vue du corps, la manière qui semble la plus facile pour que le récepteur comprenne ce que signifie ce qui lui est dit ou montré est qu'il associe d'une façon ou d'une autre sa propre production de l'événement moteur à la réception sensorielle de l'événement moteur d'autrui. Colwyn Trevarthen, qui attache beaucoup d'importance à la musicalité des corps, affirme que "Nous ressentirions instinctivement les mêmes forces et de fait, nous partagerions, par sympathie, les mêmes battements produits intérieurement et l' "intonation" de l'activité musculaire. Ainsi, nos corps seraient instinctivement reliés dans l'effectuation d'actions coordonnées vers des buts partagés » (1999-2000: 158).

La sympathie et l'empathie jouissent d'un statut tout à fait particulier. Et la définition de ces notions est disputée. Frédérique de Vignemont et Tania Singer (2006) remarquent qu'il existe presque autant de définitions de l'empathie que de chercheurs qui travaillent sur le sujet.

Le débat actuel autour du concept d'empathie est fortement marqué par le terme Einfühlung ( ressenti de l'intérieur») créé par Robert Vischer (1873) et développé par Theodor Lipps (1903-04) qui place la résonance sensorielle au cœur du lien empathique, défini comme un phénomène fondamentalement corporel. Gérard Jorland revient sur l'histoire du concept d'empathie, et conclut que l'empathie a « la structure d'une relation d'équivalence » et fonde ainsi l'intersubjectivité. Reprenant l'expression de René Thom « Le chat est la souris " Gérard Jorland affirme que "seule l'empathie permet à l'un d'anticiper le comportement de l'autre et donc de l'atteindre ou de l'esquiver » (Berthoz et Jorland, $2004: 48$ ).

Alain Berthoz distingue l'empathie de la sympathie et de la théorie de l'esprit. Dans un article récent, il définit avec Bérengère Thirioux ces deux termes en référence à l'espace: «Dans l'empathie, nous nous projetons dans 
l'autre (nous adoptons l'autre perspective) tandis que dans la sympathie nous tendons à localiser l'autre dans nous-mêmes » $(2010: 34)$. Et ils ajoutent qu'il y a concordance temporelle et que celle-ci «peut refléter un phénomène de résonance motrice, montrant que le comportement moteur d'un individu se synchronise avec le comportement de l'individu observé » (idem : 54).

Nicolas Georgieff travaille sur l'empathie en associant les réflexions menées dans le cadre des neurosciences, de la psychopathologie et de la psychanalyse. Pour lui, l'empathie correspond à l'identification à autrui parce qu'elle repose bien sur le partage de représentations ou d'expériences mais elle nécessite aussi un mécanisme de distinction de l'un et l'autre: elle pose " la problématique du même et de l'autre, du semblable et du différent, de l'ipséité et de l'altérité » (2008: 375). L'empathie est caractérisée par l'importance du phénomène de réciprocité: "L'individu cherche à se faire connaître et reconnaître de l'autre autant qu'à le connaître. Cette double nature de l'empathie semble bien à l'origine de la réciprocité des conduites » (idem:382). Un point important que soulève Nicolas Georgieff est l'effet que produit l'empathie sur l'un et l'autre : « La théorie de l'empathie définit un processus par lequel l'activité psychique de l'un est modifiée, transformée, ou influencée par celle de l'autre, parce qu'elle est conduite à reproduire partiellement, pour la partager, celle d'autrui » (ibid. : 386).

Dans le cadre de cette approche interactive - collaborative, devrais-je même dire -, Nicolas Georgieff affirme que le soi ne se constitue pas de la séparation et de sa différenciation progressive d'avec autrui mais "naît et perdure au sein d'une expérience psychique nécessairement et constamment partagée, dans une copensée et coconscience originaires » (ibid. : 369). Il est catégorique : "La subjectivité est intersubjectivité par essence, la "co-conscience " est et reste la condition de la conscience de soi » (ibid. : 369).

Des chercheurs comme Marc Jeannerod, Jean Decety ou encore Giacomo Rizzolatti ont mis en évidence l'importance de la simulation de l'action en ce qui concerne la préparation de l'action, l'observation d'autrui en train d'agir, l'imagination d'une action. La théorie de la simulation est récente et privilégie une approche par le corps plutôt que par des théories abstraites (théorie de l'esprit, par exemple). Gunther Knoblich et Natalie Sebanz (2006) travaillent sur la compréhension par l'action simulée. Celle-ci repose sur une conception de l'action humaine fondée sur des pratiques, des procédures, des savoir-faire plutôt que sur la donation de sens à l'action des autres. Ainsi, les buts et les intentions se révèlent immédiatement. L'action partagée est une modalité de la compréhension de l'action d'autrui. Jöelle Proust décrit la simulation comme un engagement dans une situation plutôt que comme un processus liant deux individus séparés. Ainsi, elle affirme que " [1]'expérience acquise par la simulation ne porte ni sur soi ni sur autrui, mais sur des situations réelles et contrefactuelles et sur leurs combinaisons. Elle a valeur de connaissance parce qu'elle donne accès à des cas de raisonnement pratique que le sujet seul n'aurait pas rencontré. » (2000:304). 


\section{LE RYTHME DES CORPS}

Toutes ces formes d'action partagées intègrent des phénomènes de synchronisation entre des individus qui sont au cœur de la fabrication de mondes communs (Cerclet, 2010).

La synchronisation se retrouve à différents niveaux de l'univers que ce soit dans les domaines de la matière, des systèmes naturels, du vivant et du social. Ici, la synchronisation est de l'ordre de l'appariement des processus et de l'organisation des rythmes qu'ils soient respiratoires, musculaires ou neuronaux. Natalie Sebanz et ses collègues (2006) déclaraient que les formes d'actions conjointes reviennent à incorporer le temps des actions des autres. Elle montrait que cette activité est plus simple que de se maintenir en état de désynchronisation. Olivier Oullier (2008) a étudié des situations de syncopation et a aussi montré que se maintenir à contre-temps nécessite un investissement énergétique plus important et une plus grande attention à la préparation et au contrôle du mouvement.

Rudolpho Llinas considère le mouvement volontaire comme une suite de tremblements : le geste serait réalisé de manière discontinue dans le temps et les opérations d'un tel système se produiraient ainsi à intervalles discrets. Le rythme du tremblement physiologique du muscle existe, inchangé dans sa périodicité de 8 à 12 hertz, indépendamment de la vitesse du mouvement et des contraintes imposées au muscle. Llinas se réfère à la prosodie non pas comme ce qui s'ajouterait au message mais comme ce qui correspondrait à la gestualisation d'un état interne. C'est une expression extérieure d'une abstraction centralement produite qui signifie quelque chose. "Pour nous, sourire, rire, froncer ou lever ses sourcils sont des formes de prosodie, par lesquelles nous communiquons un état interne et momentané, d'une manière reconnaissable et compréhensible à quelqu'un d'autre » (2001 : 229-230).

Comme nous l'avons vu tout au long de cet exposé, la compréhension et la connaissance d'autrui ou d'une situation repose sur des mécanismes de résonance qui ne peuvent être séparés de leur dimension rythmique. Le rythme est omniprésent aux niveaux perceptif, neuronal aussi bien que musculaire. Mais deux phénomènes partageant le même rythme ou la même fréquence ne font plus qu'un. Pour que nous puissions les distinguer, il importe donc que les rythmes soient produits et perçus en léger décalage.

Le rythme est l'une des dimensions de notre participation au mouvement de notre environnement. Ainsi, nous avons tous pris conscience, à un moment ou à un autre, de notre capacité à nous synchroniser avec le tempo d'une musique ou celui des applaudissements à la fin d'une représentation, avec celui d'un ou une artiste ou encore avec celui de la personne avec laquelle nous marchons, nous discutons, nous mangeons, etc. 
Pour approcher un peu plus ces mécanismes, nous devons nous poser la question de ce qu'est le rythme. Les chercheurs sont nombreux à s'être confrontés à ce concept fuyant. Lefebvre (1992) le situe dans le vécu, le charnel, le corps. Maldiney dit que « le rythme est dans les remous de l'eau, non dans le cours du fleuve " $(1973: 158)$ et Meschonnic que «le rythme est une actualisation du sujet, de sa temporalité » (1982: 87). Pour Paul Valéry, le rythme est une "relation particulièrement simple entre le percevoir et le produire », entre le potentiel et l'actuel (1973: 1336).

Emile Benvéniste a fait un important travail d'exégèse du terme rythme. Il précise que pour Démocrite, $\rho v \theta \mu \mathrm{s}$ « est toujours "forme", en entendant par là la forme distinctive, l'arrangement caractéristique des parties dans un tout » (1966: 330) et que pour Aristote, « les relations fondamentales entre les corps s'établissent par leur différences mutuelles » (idem : 328). Selon Benvéniste, « la formation en - $(\theta) \operatorname{\mu os}[\ldots]$ indique, non l'accomplissement de la notion, mais la modalité particulière de son accomplissement, telle qu'elle se présente aux yeux» (ibid. : 332). Il insiste pour dire qu'une «forme» n'est jamais fixe, réalisée, posée en quelque sorte comme un objet. Il distinguait ce $\rho v \theta \mu o s$, associé à ce qui est mouvant et fluide au $\rho v \theta \mu o s$ de Platon qui est arrangement harmonieux des attitudes corporelles combiné avec un mètre, c'est-à-dire en temps alterné (rapide et lent, par exemple). Mais le travail pointilleux de Pierre Sauvanet (1996) est venu ébranler cette distinction trop franche. Ces deux conceptions cohabitent avec intelligence et donnent ensemble une manière intéressante d'appréhender le rythme. "C'est précisément, selon Pierre Sauvanet, cette polysémie originelle du mot, cette polyvalence originelle de la chose, qui constituent l'enjeu du rythme comme concept possible: entre forme et flux, continu et discontinu, espace et temps, entre perception et création, sujet et objet, agi et subi » (idem : 31). Jean-Jacques Wunenburger met en évidence les nouvelles dimensions du rythme qui n'est pas que pure naturalité et qui possède une épaisseur, une densité, une hétérogénéité consubstantielle. Il affirme très utilement que

notre corps est moins un lieu où s'expriment des rythmes, que ce par quoi et en quoi nous connaissons les rythmes, au sens où le redoublement cognitif implique que nous soyons précisément co-acteur de la rythmicité. La rythmicité obéit donc, malgré ses apparences macro-physiques, à la même épistémologie que la microphysique contemporaine, celle de la non-séparabilité du sujet et de l'objet. (1992 : 242)

\section{CONCLUSION}

Le mouvement n'est pas un flux éthéré. Il faut compter avec la matière, qu'elle soit, selon les expressions des physiciens, à gros grains ou à grains fins. La distinction est avant tout une question de regard et de distance. Sans doute le plus difficile est de s'entendre sur la manière d'envisager cette texture. 
L'envisage-t-on dans les termes d'une transformation «quasi statique »? Ce qui revient à privilégier l'état d'équilibre. Mais nous pouvons préférer privilégier le mouvement. L'état d'équilibre n'est dès lors que transitoire. C'est « la loi d'alternance » entre mouvement et équilibre (Jullien, 1989) qui prévaut et rythme le cours du procès. La réalité va se faisant c'est-à-dire qu'elle est fondamentalement dynamique et ne peut être envisagée que de manière spatiotemporelle. Cette seconde conception me semble plus appropriée aux caractères d'un système vivant qui démontre dès ses premiers instants une capacité à entrer en relation et chercher la reconnaissance. Le présent et le maintenant ne seraient pas des états privilégiés mais de simples points de repère comme le suggère Wittgenstein. Cette approche aurait aussi l'avantage de concilier forme et rythme comme les deux dimensions des divers processus qui nous environnent. "La forme n'est pas, elle existe. [...] La forme est le rythme du matériau, qui accède par là à une existence inédite » (Maldiney, op. cit. : 163). «Le rythme c'est une forme en formation » (Sauvanet, 1999: 47). Pascal Michon affirme, dans le prolongement de la sociologie de Norbert Elias, que nous ne saisissons que "des "êtres" toujours en individuation ». Nous avons accès, selon lui, " ni [à] des ensembles d'atomes indépendants juxtaposés, ni [à] des tissus de chaînes d'interactions plus ou moins plissés, ni même [à] des configurations en déformation permanente [...] mais [à] des rythmes (au sens de formes descriptibles de réalités mouvantes) » (2005: 423).

Marcel Jousse, à travers le rythme et l'imitation, avait renoué avec un monde de nature systémique dans lequel la relation prend le dessus sur la distinction des éléments de l'ensemble. Il rompait ainsi avec le nominalisme métaphysique de Guillaume d'Ockham et inaugurait, avant l'heure, le développement de la pensée complexe dont Edgar Morin s'est fait le héraut dans le domaine des sciences humaines et sociales. Cette approche est intéressante car elle relie les hommes en un système résonant et les relie à leur environnement. Non seulement, l'être humain n'est plus un atome isolé, nostalgique d'une communauté perdue mais il renoue aussi avec la nature après en avoir été longuement séparé. Une telle pensée oblige à revoir les modalités de la relation. Jousse avait mis en place un système fondé sur la plasticité de l'être humain, capable d'absorber les vibrations du monde et de les rejouer en leur accordant collectivement une signification sociale. Le corps est au cœur de se dispositif parce qu'il est ouvert à la fois à la rythmique du cosmos et aux rythmes de l'exercice du social. Et parce qu'il est continuellement en cours d'individuation, l'individu contribue autant au façonnement d'autrui que de lui-même. Jousse disait bien à propos que l'individu ignore «ce qui va être sa substance » : il n'y a pas de soi préalable mais un continuel mouvement, qui, par les modulations introduites par la diversité des corps et des expériences, contribue à complexifier le monde. 


\section{BIBLIOGRAPHIE}

Bernard ANDRIEU, Le monde corporel de la constitution interactive de soi, Lausanne, L'Age d'Homme, 2010.

Emile BENVENISTE, "La notion de "rythme" dans son expression linguistique ", in Problèmes de linguistique générale, Paris, Editions Gallimard, vol 1, 1966, pp. 327-335.

Alain BERTHOZ, La décision, Paris, Odile Jacob, 2003.

Alain BERTHOZ et Gérard JORLAND (eds.), L'empathie, Paris, Odile Jacob, 2004.

Alain BERTHOZ et Bérangère THIRIOUX, "A spatial and perspective change theory of the difference between sympathy and empathy", Paragrana, 19/1, 2010, pp. 32-61.

Denis CERCLET, «Les corps en mouvement comme lieu de constitution du temps ? ", in Alain BERTHOZ et Bernard ANDRIEU (eds.), Le corps en acte. Centenaire Maurice Merleau-Ponty, Nancy, Presses Universitaires de Nancy, 2010, pp. 171-185.

Michael C. CORBALLIS, From hand to mouth. The Origins of Langage, Princeton, Princeton University Press, 2002.

Jean DECETY, « Naturaliser l'empathie », L'Encéphale, 28, 2002, pp. 9-20.

Norbert ELIAS, La société des individus, Paris, Librairie Arthème Fayard, 1991 pour la traduction française.

Filippo FIMIANI, «Simulations incorporées et tropismes empathiques. Notes sur la neuro-esthétique », Images Revues, 6, 2009 [En ligne]. URL: http://imagesrevues.revues.org/426

Barbara FORMIS (ed.), Penser en corps. Soma-esthétique, art et philosophie, Paris, L'Harmattan, 2009.

Shaun GALLAGHER, How the body shapes the mind, Oxford, New-York, Oxford University Press, 2005.

Nicolas GEORGIEFF, "Théorie de l'esprit et psychopathologie », La Psychiatrie de l'enfant, 48, 2, 2005 [En ligne]. URL : www.cairn.info/revue-la-psychiatrie-de-l-enfant2005-2-page-341.htm

Nicolas GEORGIEFF, «L'empathie aujourd'hui : au croisement des neurosciences, de la psychopathologie et de la psychanalyse ", La psychiatrie de l'enfant, 2, vol. 51, 2008, pp. 357-393.

Marc JEANNEROD, “The representing brain. Neural correlates of motor intention and imagery", Behavioral and Brain Sciences, 17, 1994, pp. 187-245.

Marcel JOUSSE, L'anthropologie du geste, Paris, Tel Gallimard, 1974.

François JULLIEN, Procès ou création. Une introduction à la pensée chinoise, Paris, Editions du Seuil, 1989.

Hervé KEMPF, «Entretien avec Francisco Varela », La Recherche, 308, avril 1998, pp.109-112

Günther KNOBLICH et Natalie SEBANZ, "The social nature of perception and action", Current Directions in Psychological Science, 15, 2006, pp. 99-104.

Henri LEFEBVRE, Éléments de rythmanalyse. Introduction à la connaissance des rythmes, Paris, Syllepse, 1992. 
Rodolfo R. LLINAS, I of the vortex. From neurons to self, Cambridge-London, MIT Press, 2001.

Henri MALDINEY, "L'esthétique des rythmes », in Regard, Parole, Espace, Lausanne, L'Âge d'Homme, 1973, pp.147-172.

Andrew N. MELTZOFF, "La théorie du "like me", précurseur de la compréhension sociale chez le bébé : imitation, intention et intersubjectivité », in Jacqueline NADEL et Jean DECETY (eds.), Imiter pour découvrir l'humain. Psychologie, neurologie, robotique et philosophie de l'esprit, Paris, PUF, 2002, pp. 33-57.

Maurice MERLEAU-PONTY, Phénoménologie de la perception, Paris, Gallimard, 1945, rééd. coll. Tel 1981.

Maurice MERLEAU-PONTY, Le visible et l'invisible, Paris, Gallimard, 1964, réed. coll. Tel, 2006.

Henri MESCHONNIC, Critique du rythme. Anthropologie historique du langage, Lagrasse, Verdier, 1982.

Pascal MICHON, Rythmes, pouvoir, mondialisation, Paris, PUF, 2005.

Alva NOÉ, Action in perception, Cambridge, London, The MIT Press, 2005.

Olivier OULLIER, J. A. SCOTT KELSO et Alan P. KIRMAN, "Social Neuroeconomics: A dynamical systems perspective", Revue d'économie politique, $\mathrm{n}^{\circ} 1$, vol. 118, 2008, pp. 51-62.

Joëlle PROUST, "Pour une théorie "motrice" de la simulation », in Jean DECETY (ed.), Cerveau, perception et action, Psychologie française, déc. 2000, T. 45 - 4, pp. 295306.

Arnaud REVEL et Jacqueline NADEL, "How to build an imitator", in Chrystopher L. NEHANIV and Kerstin DAUTENHAN (eds.), Imitation and Social Learning in Robots, Humans and Animals: Behavioural, Social and Communicative Dimensions, Cambridge University Press, 2007, pp. 279-300.

Giacomo RIZZOLATTI et Corrado SINIGAGLIA, Les neurones miroirs, traduit de l'italien par M. Raiola, Paris, Ed. Odile Jacob, 2008.

Pierre SAUVANET et Jean-Jacques WUNENBURGER (dir.), Rythmes et Philosophie, Paris, Editions Kimé, 1996.

Pierre SAUVANET, Le Rythme grec d'Héraclite à Aristote, Paris, PUF, 1999.

Natalie SEBANZ, Harold BEKKERING et Günther KNOBLICH, “Joint action: bodies and minds moving together", TRENDS in Cognitive Sciences, Vol.10, no.2 February 2006, pp. 70-76.

Richard SHUSTERMAN, Sous l'interprétation, Paris, Editions de l'Eclat. Coll. Tiré à part, 1994.

Richard SHUSTERMAN, Conscience du corps. Pour une soma-esthétique, Traduction de N. Vieillescazes, Paris, Editions de l'Eclat. Coll. Tiré à part, 2007.

Juliette SOULEZ, «Du Pluralisme au Méliorisme, de L'expérience au Corps : Richard Shusterman", Tale(s) Magazine, no. 5, [en ligne]. URL: http://www.talesmagazine.fr/light-online-mag/polygon\#article204_item109.

Colwyn TREVARTHEN, "Musicality and the intrinsic motive pulse: evidence from human psychobiology and infant communication", in Rhythms, Musical Narrative, 
and the Origins of Human Communication, Musicae Scientiae, Special Issue, 19992000, pp. 157-213.

Francisco VALERA, Evan THOMPSON et Eleanor ROSCH, The embodied mind: cognitive science and human experience, Cambridge, The MIT Press, 1991.

Paul VALERY, Cahiers, tome 1, Paris, NRF, Bibliothèque de la Pléiade, 1973.

Frédérique de VIGNEMONT et Tania SINGER, "The empathic brain: how, when and why?", TRENDS in Cognitive Sciences, Vol.10, No.10, 2006 [en ligne]. URL: http://hal.archives-ouvertes.fr/docs/00/16/95/84/PDF/empathy_TICS.pdf

Jean-Jacques WUNENBURGER (dir.), Les Rythmes. Lectures et théories, Paris, L'Harmattan, 1992.

RÉSUMÉ : L'objectif de cet article est de revenir sur l'édifice construit par Marcel Jousse à l'articulation des sciences sociales et biologiques. Il s'agit de montrer toute l'actualité de ce modèle qui associe l'individu, autrui et leur environnement en le resituant dans une filiation théorique qui tient le corps en mouvement et en relation pour fondement à la fois du social et des processus d'individuation.

MOTS-CLÉS : Jousse, corps, geste, résonance, synchronisation, rythme, anthropologie, neurosciences. 